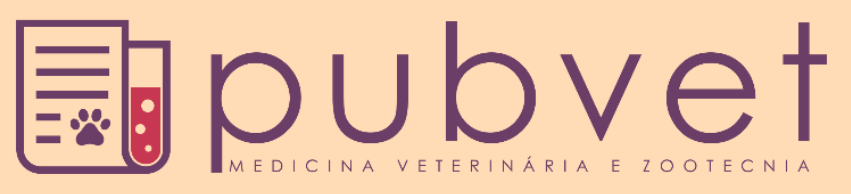

https://doi.org/10.31533/pubvet.v12n10a183.1-5

\title{
Estudo etnoveterinário de plantas medicinais utilizadas em animais da microrregião do Alto Médio Gurguéia - Piauí
}

\author{
Wenderson Rodrigues de Amorim ${ }^{1 *} \bullet$, Caike Pinho de Sousa ${ }^{1}{ }^{\bullet}$, Gabriel do Nascimento \\ Martins $^{1}{ }^{\ominus}$, Eveny Silva de Melo ${ }^{1}{ }^{\ominus}$, Italo Carlos Rodrigues da Silva ${ }^{1}{ }^{\circ}$, Paulo Gomes do \\ Nascimento Corrêa ${ }^{2} \bullet$, Alan Rodrigo Sousa Soares $\operatorname{Santos}^{2}{ }^{\circ}$, Sávio Matheus Reis de \\ Carvalho $^{3}$, Raizza Eveline Escórcio Pinheiro ${ }^{4}{ }^{\bullet}$, Jamylla Mirck Guerra de Oliveira ${ }^{4} \bullet$ \\ ${ }^{I}$ Graduado em Medicina Veterinária pela Universidade Federal do Piauí, Bom Jesus - PI, Brasil.

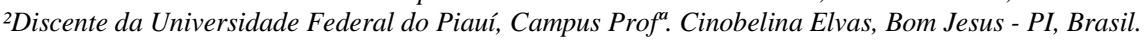 \\ ${ }^{3}$ Residente em Medicina Veterinária pela Universidade Federal do Piauí, Campus Teresina - PI, Brasil. \\ ${ }^{4}$ Docente da Universidade Federal do Piauí, Departamento de Medicina Veterinária, Bom Jesus-PI, Brasil. E-mail: jamyllaguerra@ gmail.com \\ *Autor para correspondência, E-mail: rodriguesamorim10@gmail.com
}

\begin{abstract}
RESUMO. Objetivou-se com o trabalho investigar, resgatar e documentar o conhecimento popular sobre a utilização de plantas medicinais na promoção da saúde animal, na microrregião do Alto Médio Gurguéia, Piauí. Foram selecionadas como entrevistados pessoas com experiência na criação de animais, e com conhecimentos sobre utilização de plantas medicinais, empregando amostragem não aleatória. Para isso realizou-se visitas a propriedades rurais, e a agências de apoio aos produtores rurais (ADAPI e EMATER). Foram realizadas 61 entrevistas entre produtores e profissionais das ciências agrárias, sem distinção de sexo ou idade. Para as entrevistas, foram utilizados formulários semiestruturados com perguntas abertas e fechadas. Durante a pesquisa de campo, foram citadas 43 espécies de plantas utilizadas no tratamento de doenças ou afecções animais. Apenas as seis mais citadas estão descritas em tabela, Aloe Vera L., Drimys brasiliensis, Poincianella pyramidalis (Tul.), Chenopodium ambrosioides L., Gossypium hirsutum, Caryocar coriaceum Wittm, Hancornia speciosa Gomez, Guapira graciliflora, Mauritia flexuosa, abordando detalhes sobre a taxonomia, indicações terapêuticas, as partes utilizadas, as maneiras de preparo e os animais tratados. As espécies descritas foram as mais citadas não por coincidência, são plantas que se destacam em diversas pesquisas etnobotânicas e demonstram uma ampla potencialidade medicinal, mesmo que possuam escassas comprovações científicas de suas atividades biológicas.
\end{abstract}

Palavras chave: Aloe vera, conhecimento popular, etnobotânica, saúde animal

\section{Ethnoveterinary study of medicinal plants used in animals from the microregion of Alto Médio Gurguéia - Piauí}

ABSTRACT. The objective of this work was to investigate, rescue and document popular knowledge about the use of medicinal plants in the promotion of animal health in the Alto Médio Gurguéia microregion, Piauí. We interviewed people with experience at animal husbandry, and with knowledge about the use of medicinal plants, using non-random sampling. For this purpose, visits were made to rural properties and to support agencies for rural producers (ADAPI and EMATER). There were 61 interviews were conducted between producers and professionals in the agrarian sciences, regardless of sex or age. For the interviews, semi-structured forms with open and closed questions were used. During field research 43 species of plants used in the treatment of animal diseases or conditions were cited. Only at six most cited are described in the table, Aloe Vera L., Drimys 
brasiliensis, Poincianella pyramidalis (Tul.), Chenopodium ambrosioides L., Gossypium hirsutum, Caryocar coriaceum Wittm, Hancornia speciosa Gomez, Guapira graciliflora, Mauritia flexuosa, detailing the taxonomy, therapeutic indications, the parts used, the methods of preparation and the treated animals. The species described were the most cited not by coincidence, are plants that stand out in several ethnobotanical researches and demonstrate a wide medicinal potentiality, even though they have scarce scientific evidence of their biological activities.

Keywords: Aloe vera, popular knowledge, ethnobotany, animal health

\title{
Estudio etnoveterinario sobre plantas medicinales utilizadas en animales de la microrregión del Alto Medio Gurguéia - Piauí
}

\begin{abstract}
RESUMEN. Se objetivó con este trabajo investigar, rescatar y documentar el conocimiento popular sobre la utilización de plantas medicinales en la promoción de la salud animal, en la microrregión del Alto Medio Gurguéia, Piauí. Se seleccionaron como entrevistadas personas con experiencia en la cría de animales, y con conocimientos sobre el uso de plantas medicinales, empleando muestreo no aleatorio. Para ello se realizaron visitas a propiedades rurales y agencias de apoyo a los productores rurales (ADAPI y EMATER). Se realizaron 61 entrevistas entre productores y profesionales de las ciencias agrarias, sin distinción de sexo o edad. Para las entrevistas, se utilizaron formularios semiestructurados con preguntas abiertas y cerradas. Durante la investigación de campo, fueron citadas 43 especies de plantas utilizadas en el tratamiento de enfermedades o afecciones animales. Sólo las seis más citadas se describen en tabla, Aloe Vera L., Drimys brasiliensis, Poincianella pyramidalis (Tul.), Chenopodium ambrosioides L., Gossypium hirsutum, Caryocar coriaceum Wittm, Hancornia speciosa Gomez, Guapira graciliflora, Mauritia flexuosa, abordando detalles sobre la taxonomía, indicaciones terapéuticas, las partes utilizadas, las formas de preparación y los animales tratados. Las especies descritas fueron las más citadas no por coincidencia, son plantas que se destacan en diversas investigaciones etnobotánicas y demuestran una amplia potencialidad medicinal, aunque tengan escasas comprobaciones científicas de sus actividades biológicas.
\end{abstract}

Palabras chave: Aloe vera, conocimiento popular, etnobotánica, salud animal

\section{Introdução}

O termo etnoveterinária utilizado pela primeira vez em 1980 por McCorkle é uma área da ciência que aborda a opinião, conhecimento e as práticas populares direcionadas a promoção da saúde animal, portanto, um dos seus métodos consiste na fitoterapia, ou seja, a utilização de plantas medicinais no tratamento de enfermidades animal (Teixeira et al., 2015).

No Brasil, o desenvolvimento da fitoterapia está ligado essencialmente as influências culturais indígenas, africanas e europeias. As práticas e conhecimentos adquiridos ainda são utilizados por diversos produtores, fazendeiros e médicos veterinários na intenção de tratar as moléstias do rebanho e dos animais de companhia (Monteiro et al., 2012).

$\mathrm{O}$ aumento de gastos com medicamentos e serviços veterinários, a dificuldade de encontrar fármacos convencionais, a ausência de assistência técnica veterinária, além de exigências do mercado por produtos naturais tem contribuíndo para o crescimento dessa atividade, ao passo que, é iminente o aumento no interesse em estudar-se ainda mais a área (Chagas et al., 2008).

A etnoveterinária por ser uma ciência popular, divulgada verbalmente entre gerações, ao longo de muitos anos, nos remete a pensar na necessidade de documentar esse tipo de conhecimento, para evitar que, com o passar dos anos, devido as grandes mudanças sociais, culturais e ecológicas, essa prática tão importante seja perdida.

Este trabalho foi realizado para investigar, resgatar e documentar o conhecimento popular sobre a utilização de plantas medicinais na promoção da saúde animal, na microrregião do Alto Médio Gurguéia, Piauí.

\section{Material e Métodos}

A coleta de dados foi realizada na microrregião do Alto médio Gurguéia - Piauí nos meses de maio e junho de 2017, em entrevistas individuais, 
envolvendo pessoas e propriedades de oito municípios (Bom Jesus, Currais, Santa Luz, Cristino Castro, Palmeira do Piauí, Redenção do Gurgueia, Gilbués, Alvorada do Gurgueia). Foram selecionadas como entrevistados pessoas com experiência na criação de animais e com conhecimentos sobre utilização de plantas medicinais, empregando amostragem não aleatória. Para isso realizou-se visitas a propriedades rurais, e a agências de apoio aos produtores rurais (ADAPI e EMATER). Foram realizadas 61 entrevistas entre produtores e profissionais das ciências agrárias, sem distinção de sexo ou idade. Para as entrevistas foram utilizados formulários semiestruturados com perguntas abertas e fechadas, modelo adaptado de Morais et al. (2005). Antes da aplicação dos formulários, foi esclarecido para os entrevistados todos os objetivos do trabalho e, somente após obtenção do consentimento verbal, e da assinatura em duas vias do Termo de consentimento livre e esclarecido, as entrevistas iniciaram. O termo garante que os direitos do participante serão respeitados. As perguntas tratavam sobre as espécies, enfermidades e a forma que os agricultores utilizam as plantas medicinais para o tratamento dos seus animais.
Os dados coletados foram organizados por meio do Microsoft Excel 2016, analisados quantitativamente e apresentados em tabela as plantas mais utilizadas entre os entrevistados, de maneira descritiva.

\section{Resultados e Discussão}

Durante a pesquisa de campo foram citadas 43 espécies de plantas utilizadas no tratamento de doenças ou afecções animais. Apenas as seis espécies mais citadas são apresentadas na tabela 1 , abordando detalhes sobre a taxonomia, indicações terapêuticas, as partes vegetais utilizadas e as maneiras de preparo. Em relação aos animais doentes, de acordo com os entrevistados, os tratamentos descritos se aplicam a todas as espécies dos animais domésticos.

A Babosa (Aloe vera L.) foi utilizada principalmente no tratamento de feridas infeccionadas, e como cicatrizante. Segundo Lawrence et al. (2009), o que confere esse potencial à planta está relacionado a atividade antimicrobiana proporcionada essencialmente pelas antraquinonas presentes em seu arranjo químico estrutural, além disso, ainda possui em sua composição o tanino, que auxilia no processo granulomatoso e contração da ferida.

Tabela 1. Plantas mais utilizadas e sua indicação popular no tratamento de doenças de animais domésticos da Microrregião Alto Médio Gurguéia, Piauí.

\begin{tabular}{|c|c|c|c|c|}
\hline Nome Científico & Nome popular & Parte usada & Forma de uso & Indicação \\
\hline Aloe Vera L. & Babosa & Folhas & $\begin{array}{l}\text { Cataplasmo, chá, "in } \\
\text { natura" }\end{array}$ & $\begin{array}{l}\text { Ferimentos infeccionados, } \\
\text { Cicatrizante, Energético, } \\
\text { Estimulante do apetite; Diarreia, } \\
\text { Retenção de anexos fetais. }\end{array}$ \\
\hline Drimys brasiliensis & Pra-tudo & $\begin{array}{l}\text { Casca e } \\
\text { entrecasca }\end{array}$ & Decocção, maceração & $\begin{array}{l}\text { Retenção de anexos fetais, "mal dos } \\
\text { chifres em bovinos". }\end{array}$ \\
\hline $\begin{array}{l}\text { Poincianella } \\
\text { pyramidalis (Tul.) }\end{array}$ & $\begin{array}{l}\text { Catinga de } \\
\text { Porco }\end{array}$ & $\begin{array}{l}\text { Casca, } \\
\text { entrecasca e } \\
\text { folhas }\end{array}$ & $\begin{array}{l}\text { Decocção, maceração } \\
\text { e cataplasmo }\end{array}$ & $\begin{array}{l}\text { Diarreia bovina, Ferimentos com } \\
\text { Miíase, Cicatrizante. }\end{array}$ \\
\hline $\begin{array}{l}\text { Chenopodium } \\
\text { ambrosioides } \mathrm{L} .\end{array}$ & Mastruz & $\begin{array}{l}\text { Folhas e } \\
\text { caule }\end{array}$ & $\begin{array}{l}\text { Cataplasma, } \\
\text { Maceração e } \\
\text { unguento }\end{array}$ & $\begin{array}{l}\text { Luxações, Fraturas, Afecções e } \\
\text { Infecções cutâneas, Verminose e } \\
\text { estimulante do apetite. }\end{array}$ \\
\hline Gossypium hirsutum & Algodão & Folhas & Chá, maceração & $\begin{array}{l}\text { Retenção de anexos fetais, fraturas } \\
\text { e diarreia. }\end{array}$ \\
\hline $\begin{array}{l}\text { Caryocar coriaceum } \\
\text { Wittm }\end{array}$ & Pequi & Folhas & Chá, maceração & Retenção de anexos fetais \\
\hline $\begin{array}{l}\text { Hancornia speciosa } \\
\text { Gomez }\end{array}$ & Mangabeira & $\begin{array}{l}\text { Folhas, } \\
\text { casca, } \\
\text { entrecasca e } \\
\text { raiz }\end{array}$ & $\begin{array}{l}\text { Cataplasma, Chá, } \\
\text { maceração, decocção }\end{array}$ & $\begin{array}{l}\text { Lesão de pele, Ferimentos com } \\
\text { miíases, cicatrizante e diarreia. }\end{array}$ \\
\hline Guapira graciliflora & Pau-piranha & $\begin{array}{l}\text { Casca e } \\
\text { entrecasca }\end{array}$ & $\begin{array}{l}\text { Decocção e } \\
\text { maceração }\end{array}$ & Retenção de anexos fetais \\
\hline Mauritia flexuosa & Buriti & Fruto & Óleo & $\begin{array}{l}\text { Cicatrizante, anti-inflamatório, } \\
\text { acidente ofídico. }\end{array}$ \\
\hline
\end{tabular}


Para Marshall (1990), a Babosa possuí ação bactericida devido aos açucares presentes, que desempenham o aumento da pressão osmótica deixando o ambiente hostil as bactérias. Além disso, sugere que o lactato de magnésio presente na planta seja responsável pela redução na liberação de histamina na resposta inflamatória o que favorece o processo de recuperação tecidual. A Babosa é útil na manutenção homeostática do organismo, devido seu potencial energético, representado por sua composição repleta de polissacarídeos, carboidratos, aminoácidos essenciais, minerais e vitaminas (Freitas et al., 2014). Porém, Dunnick \& Nyska (2013) destacam que é necessário utilizá-la com moderação, principalmente nos problemas gastrointestinais, pois o uso prolongado da planta pode causar efeitos laxativos e até neoplásicos, devido as quinonas, que depois de metabolizadas possuem potencial cancerígeno no intestino.

Assim como a Babosa, a Catinga de porco e a Mangabeira estiveram entre as plantas mais utilizadas no tratamento de feridas abertas, ou seja, com alto potencial cicatrizante. A primeira bem comentada na literatura, onde são realizados uso de infusão e decoctos, obtidos a partir de todas as partes da planta, direcionados ao tratamento de diversas enfermidades, principalmente doenças infecciosas e inflamatórias. De acordo com estudos fitoquímicos, a principal substância ativa na planta são os compostos fenólicos (Ribeiro et al., 2014; Silva et al., 2015). Em um levantamento realizado sobre as plantas populares mais utilizadas pela comunidade de São Cristóvão - SE, também se constatou que o látex da mangabeira era bastante utilizado no tratamento de doenças fúngicas e bacterianas, principalmente no que diz respeito a ferimentos abertos na pele (Santos et al., 2007).

O Mastruz e o Algodão foram as únicas plantas descritas para tratamento de problemas ósseos/articulares. De acordo com Ribeiro et al. (2014), em estudo realizado em Assaré - CE a Chenopodium ambrosinoides e Gossypium hirsutum são comumente utilizadas no tratamento de reumatismo, fraturas e inflamações. Além disso, o mastruz foi unanimidade no tratamento de verminoses, principalmente em espécies caprinas e ovinas. Tais dados corroboram com Hammond et al. (1997) na qual o óleo da Chenopodium ambrosioides é usado contra Toxocara canis em cachorros. De acordo com Chagas et al. (2008) o mastruz é uma das principais alternativas para o controle de verminoses em caprinos e ovinos, sendo uma das plantas mais investigadas no Brasil quanto a sua ação helmíntica.

As plantas como Babosa, Algodão, Pra-tudo, Pequi e Pau-piranha foram bastantes citadas nos casos de retenção placentária, principalmente na espécie bovina. Esse tipo de situação é rotineira entre os criadores, e muitos buscam nessas plantas a solução para o seu problema. O sumo das folhas de Algodão e Pequi é muito utilizado por raizeiros do estado de Minas Gerais no tratamento de problemas uterinos, as plantas são responsáveis por aumentar a motilidade do órgão (Grandi et al., 1989). Popularmente, o Pra-tudo e Pau-piranha, é internamente utilizada como estimulante, antiespamodica, antidiarreica, contra sangramentos uterinos e em afeções geniturinárias (Simões, 1998; Wink, 2015).

O óleo do fruto do buriti foi bem descrito tanto na utilização por via oral e por via tópica, utilizado nas diferentes espécies, com ação cicatrizante e anti-inflamatória. Além disso, em animais que foram picados por cobra venenosa. Em estudo desenvolvido por Batista et al. (2012) constatouse que a pomada a base de Buriti, tem efeitos positivos em ratos com lesões cutâneas, devido a presença de carotenoides, vitaminas A e E que atuam na reparação tecidual. Além disso, o óleo do seu fruto tem função de reparar e lubrificar a camada hidro lipídica da pele, fundamental para uma pele saudável e rejuvenescida. Sampaio \& Carrazza (2012) acreditam também que o óleo de buriti tenha atividade antiofídica, corroborando com os dados da pesquisa. Todavia, na literatura ainda é pouco descrita, portanto, tal fato ainda deve ser melhor elucidado.

\section{Conclusão}

O estudo etnoveterinário sobre a utilização de plantas medicinais na promoção da saúde animal, desenvolvido da Microrregião do Alto Médio do Gurguéia, Piauí apresentou um notável conhecimento em relação as propriedades terapêuticas das espécies citadas. A maioria demonstra uma ampla potencialidade medicinal, mesmo que ainda possuam escassas comprovações científicas de suas atividades biológicas. As espécies Aloe vera, Drimys brasiliensis e Poincianella pyramidalis (Tul.) foram as mais citadas, e não por coincidência, são plantas que se destacam em diversas pesquisas etnobotânicas e podem ser promissoras em estudos de bioprospecção. Além do mais, o trabalho conseguiu alcançar seu objetivo central, 
investigou, resgatou e documentou as técnicas naturais de promoção da saúde animal, fazendo uma correlação muito importante entre o saber tradicional e científico.

\section{Referências}

Batista, J. S., Olinda, R. G., Medeiros, V. B., Rodrigues, C. M. F., Oliveira, A. F., Paiva, E. S., Medeiros, A. C. 2012. Atividade antibacteriana e cicatrizante do óleo de buriti Mauritia flexuosa L. Revista Ciência Rural, 42, 136-141.

Chagas, A. C. S., Vieira, L. S., Freitas, A. R., Araújo, M. R. A., Araújo-Filho, J. A., Araguão, W. R., \& Navarro, A. M. C. 2008. Anthelmintic efficacy of neem (Azadirachta indica A. Juss) and the homeopathic product Fator Vermes ${ }^{\circledR}$ in Morada Nova sheep. Veterinary Parasitology, 151(1), 68-73.

Dunnick, J. K., \& Nyska, A. 2013. The toxicity and pathology of selected dietary herbal medicines. Toxicologic Pathology, 41(2), 374386.

Freitas, V. S., Rodrigues, R. A. F., \& Gaspi, F. O. G. 2014. Propriedades farmacológicas da Aloe vera (L.) Burm. f. Revista Brasileira de Plantas Medicinais, 16(2), 299-307.

Grandi, T. S. M., Trindade, J. A., Pinto, M. J. F., Ferreira, L. L., \& Catella, A. C. 1989. Plantas medicinais de Minas Gerais, Brasil. Acta Botanica Brasilica, 3(2), 185-224.

Hammond, J. A., Fielding, D., \& Bishop, S. C. 1997. Prospects for plant anthelmintics in tropical veterinary medicine. Veterinary Research Communications, 21(3), 213-228.

Lawrence, R., Tripathi, P., \& Jeyakumar, E. 2009. Isolation, purification and evaluation of antibacterial agents from Aloe vera. Brazilian Journal of Microbiology, 40(4), 906-915.

Marshall, J. M. 1990. Aloe vera gel: what is the evidence. The Pharmaceutical Journal, 244, 360-362.

Monteiro, M. V. B., Rodrigues, S. T., \& Vasconcelos, A. L. F. 2012. Plantas medicinais utilizadas na medicina etnoveterinária praticada na ilha do Marajó. Embrapa Amazônia Oriental, 380, 1-33.
Morais, S. M., Dantas, J. D. a. P., Silva, A. R. A., \& Magalhães, E. F. 2005. Plantas medicinais usadas pelos índios Tapebas do Ceará. Brazilian Journal of Pharmacognosy, 15(2), 169-177.

Ribeiro, D. A., Oliveira, L. G. S., Macêdo, D. G., Menezes, I. R. A., Costa, J. G. M., Silva, M. A. P., Souza, M. M. A. 2014. Promising medicinal plants for bioprospection in a Cerrado area of Chapada do Araripe, Northeastern Brazil. Journal of Ethnopharmacology, 155(3), 15221533.

Sampaio, M. B., \& Carrazza, L. R. 2012. Manual tecnológico de aproveitamento integral do fruto e da folha do Buriti. Manual Tecnológico - ISPN, 4, 1-80.

Santos, P. O., Barbosa Júnior, A. M., Mélo, D. L. F. M., \& Trindade, R. C. 2007. Investigação da atividade antimicrobiana do látex da mangabeira (Hancornia speciosa GOMES). Revista Brasileira de Plantas Medicinais, 9, 108-111.

Silva, C. G., Marinho, M. G. V., Lucena, M. F. A., \& Costa, J. G. M. 2015. Levantamento etnobotânico de plantas medicinais em área de Caatinga na comunidade do Sítio Nazaré, município de Milagres, Ceará, Brasil. Revista Brasileira de Plantas Medicinais, 17(1), 133142.

Simões, C. u. M. O. 1998. Plantas da medicina popular no Rio Grande do Sul (Vol. 1). Porto Alegre, Rio Grande do Sul, Brasil: Editora da Universidade Federal do Rio Grande do Sul.

Teixeira, V. H. S., Fernandes, N. C. F., Corrêa, F. C., \& Oliveira, D. M. C. 2015. Plantas medicinas na etnoveterinária no Brasil - Artigo de revisão. Anclivepa, 42, 926-930.

Wink, M. 2015. Modes of action of herbal medicines and plant secondary metabolites. Medicines, 2(3), 251-286.

Recebido: 28 Agosto, 2018

Aprovado: 20 Setembro, 2018

Publicado: 2 Outubro 2018

Licenciamento: Este artigo é publicado na modalidade Acesso Aberto sob a licença Creative Commons Atribuição 4.0 (CC-BY 4.0), a qual permite uso irrestrito, distribuição, reprodução em qualquer meio, desde que o autor e a fonte sejam devidamente creditados. 